\title{
UNIVERSAL VARIATIONS OF HODGE STRUCTURE AND CALABI-YAU-SCHOTTKY RELATIONS
}

\author{
ZIV RAN
}

\begin{abstract}
Varieties generally come to life through the maps between them and the modules (e.g. functions) that live on them. Moduli spaces are no exception to this rule. An important class of maps on moduli spaces (for compact Kähler manifolds) is that of period maps, which are substantially the same thing as the modules known as variations of Hodge structure.
\end{abstract}

The purpose of this paper is twofold. First, we give a canonical formula for the variation of Hodge structure associated to the m-th order universal deformation of an arbitrary compact Kähler manifold without vector fields. Second, we specialize to the case of a Calabi-Yau manifold $X$ where we give a formula for the m-th differential of the period map of $X$ and deduce formal defining equations for its image (Schottky relations); these are (necessarily infinite, in dimension $\geq 3$ ) power series in the middle cohomology.

We will use the method of canonical infinitesimal deformations, developed by the author in earlier papers $[\mathrm{R} 1, \mathrm{R} 2]$. This method gives a canonical description of infinitesimal moduli spaces and, what's more, natural maps involving them. While it might be argued that a germ of a smooth space-such as the moduli of an unobstructed manifold-is a rather rigid featureless object, making a canonical description of it uninteresting, on the contrary maps involving such germs can be quite interesting; in the case of moduli, the method of canonical infinitesimal deformations provides a vehicle for studying such maps. For instance in the case 
at hand the $m$-th derivative of the period map of Calabi-Yau $n$-fold $X$ is a filtered map

$$
T_{X}^{m} M \rightarrow H_{D R}^{n}(X) / F^{n}
$$

whose associated gradeds $S^{i} H^{1}\left(T_{X}\right) \rightarrow H_{X}^{n-i, i}$ are the so-called Yukawa- Green forms (cf. [G]). We will develop cohomological formulas for this and other derivative maps (Theorems 4.1, 4.3 below), which will allow us to determine their image and derive (Schottky) relations defining this image, essentially in terms of some generalized Yukawa-Green type forms (Theorem 4.1, Corollary 4.4). For $n=2$ we recover the celebrated 'period quadric' of $K 3$ surface theory; for $n \geq 3$ the relations seem to be new. For $n=3$ this situation is particularly interesting assuming the 'mirror conjecture' because then the higher derivatives of the period map, here computed, are related -in fact, carry equivalent information to- the 'quantum cohomology'(esp. numbers of rational curves, etc.) of the mirror of $X$. We hope to return to this in greater detail elsewhere.

The present methods should be applicable in other Schottky-type problems: the case of curves is being developed by G. Liu ( UCR dissertation).

This paper is a revised version of a manuscript entitled 'linear structure on Calabi-Yau moduli spaces' (May 1993). We are grateful to Professors P. Deligne and M. Green for their enlightening comments. We are especially grateful to the referee, for his dedication above and beyond the call of duty, and for his detailed and insightful comments which have greatly improved this paper.

\section{Modular COALGebra}

In $[\mathrm{R} 2]$ we gave a characterization of the dual vector space of an artin local $\mathbb{C}$ algebra as a so-called OS (Order-Symbolic) structure. This characterization was 
used in constructing the base ring of the universal formal deformation (e.g. of a compact complex manifold). Now as might be expected, an important role in the geometry of moduli spaces, both local and global, is played by certain canonical 'modular' sheaves and modules over them which are naturally associated to the deformation problem. Thus motivated we now extend the aforementioned dual characterization from algebras to modules. The latter give rise on the other, ' coalgebra' side to a so-called Modular Order-Symbolic or MOS structure. An important guiding principle to keep in mind is that the process of passing from a module $E$ to the associated MOS structure $B(E)$ should involve no dualising, i.e. be covariant functorial in $\mathrm{E}$, because we shall want to apply this when $E$ is a cohomology group and cohomology and dualising don't mix. Thus while the algebra-OSS correspondence is essentially a matter of brute-force arrow reversal, the same is not true for module-MOSS.

Now let $\left(R_{n}, m_{n}\right)$ be an artin local $\mathbb{C}$-algebra of exponent $n$ (which to us means $m_{n}^{n+1}=0 \neq m_{n}^{n}$ ) and $V=V^{n}=m_{n}^{*}$ the associated ( standard) OS structure. It will be convenient also to consider

$$
V_{0}=V_{0}^{n}=R_{n}^{*}=V \oplus V_{0}^{0}
$$

where $V_{0}^{0}=\mathbb{C} 1^{*}$ and $V_{0}^{i}=V^{i} \oplus V_{0}^{0}, i \geq 1$ ( which might be called an augmented OSS). Given these data, a $V$-compatible MOS structure $B$ consists by definition of a filtered vector space

$$
B^{0} \subset \cdots \cdots \subset B^{n}=B,
$$

together with a set of symbol maps

$$
\sigma_{B}^{i}: B^{i} \rightarrow B^{i} / B^{0} \rightarrow V^{i} \otimes_{\mathbb{C}} B^{i-1}
$$


satisfying the obvious 'comodule' rule, which amounts to commutativity of

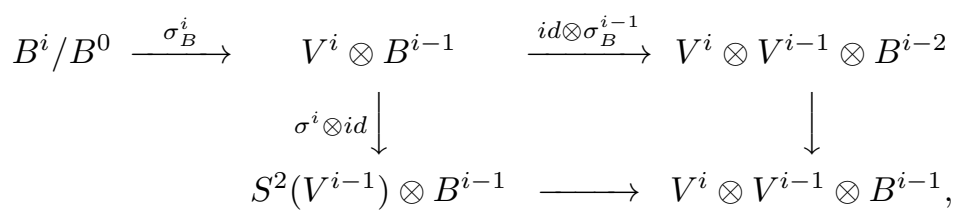

$\sigma^{i}=$ symbol map for $V^{i}$. A morphism of MOS structures is defined in the obvious way.

Example Let E be an $R_{n}$-module and set

$$
B^{i}(E)=B_{R_{n}}^{i}(E)=V_{0}^{i} \otimes_{R_{n}} E, i=1, \cdots n, B(E)=B^{n}(E)
$$

(note indeed that $V_{0}^{i}$, besides being an OS structure, is also an $R_{n}$-module by the obvious rule $(r . f)(x)=f(r x))$, with symbol map induced from the comultiplication map

$$
V_{0}^{i} \rightarrow V^{i} \rightarrow V^{i} \otimes V_{0}^{i}
$$

dual to $m \otimes_{\mathbb{C}} R \rightarrow m$, by tensoring with $E$. The assignment

$$
E \longmapsto B(E)
$$

is a covariant functor from R-modules to $V$-compatible MOS structures.

Now it is important to be able to go the other way. To this end define, for an MOS structure B,

$$
C(B)=C^{n}(B)=\operatorname{Hom}_{M O S}\left(V_{0}^{n}, B\right) .
$$

It is easy to check that the $R_{n}$-module structure on $V_{0}$ induces one on $C(B)$, and that $C$ yields a functor from MOS structures to $R$-modules.

We summarise some relevant properties of these functors as follows. 
Proposition 1.1. (i) $B$ is right exact and $C$ is left exact;

(ii) there are natural maps

$$
E \rightarrow C \cdot B(E), \quad B \cdot C(D) \rightarrow D,
$$

which are isomorphisms whenever $E$ is free (resp. $D$ is 'cofree', i.e. a sum of copies of $V_{0}$.

Proof. (i) is clear from the usual exactness properties of Hom and $\otimes$. As for (ii), the maps are defined by

$$
\begin{gathered}
e \longmapsto(v \longmapsto v \otimes e) \in \operatorname{Hom}_{M O S}\left(V_{0}, V_{0} \otimes E\right), \\
v \otimes \phi \longmapsto \phi(v), \phi \in \operatorname{Hom}_{M O S}\left(V_{0}, D\right) .
\end{gathered}
$$

It is easy to check that these are well-defined etc. To complete the proof it suffices to prove that

$$
\operatorname{Hom}_{M O S}\left(V_{0}, V_{0}\right) \simeq R \text {. }
$$

more precisely that the map $R \longmapsto \operatorname{Hom}_{M O S}\left(V_{0}, V_{0}\right), r \longmapsto r I$, is a isomorphism. It is easy to see that this map is injective. For surjectivity we argue by induction. Take $\phi \in \operatorname{Hom}_{M O S}\left(V_{0}, V_{0}\right)$. By induction there exists $r \in R_{n}$ inducing the same map as $\phi$ on $V_{0}^{n-1}$; using compatibility with comultiplication it is easy to see that $r$ and $\phi$ also induce the same map on $V=V_{0} / V_{0}^{0}$, and consequently $\phi-r I$ is effectively a map $V_{0}^{n} / V_{0}^{n-1} \rightarrow V_{0}^{0}$, i.e. given by an element of $\operatorname{Hom}_{\mathbb{C}}\left(m_{n}^{n *}, \mathbb{C}\right)=m_{n}^{n}$, say $s$. Then $\phi=(r+s) I$.

Remark 1.2 Predictably the category of $V$ - MOS also admits internal Hom and tensor product. For example if $B_{1}, B_{2}$ are $V$ - MOS we may define an MOS $C=$ 
$B_{1} \otimes_{V} B_{2}$ inductively by $C^{0}=B_{1}^{0} \otimes B_{2}^{0}$ (all tensor products over $\mathbb{C}$ unless otherwise mentioned) and setting $C^{i}$ to be the preimage of $V^{i} \otimes C^{i-1}$ by the natural map

$$
B_{1}^{i} \otimes B_{2}^{i} \rightarrow V^{i} \otimes V^{i} \otimes B^{i-1} \otimes B^{i-1}
$$

(where inductively $V^{i} \otimes C^{i-1}$ sits naturally in $V^{i} \otimes V^{i} \otimes B^{i-1} \otimes B^{i-1}$ via (comultiplication) $\otimes($ inclusion)).

Similarly we may define $\operatorname{sym}_{V}^{j}(B)$ and $\operatorname{sym}_{V}(B)$, the 'symmetric coalgebra' (or OSS) on $B$, which naturally admits an OS structure coming from the natural maps

$$
\operatorname{sym}_{V}^{i}(B) \rightarrow \sum_{j} \operatorname{sym}_{V}^{j}(B) \otimes_{V} \operatorname{sym}_{V}^{i-j}(B) .
$$

\section{SubSet SPACES AND SOME COMPleXes ON Them}

Recall that, for a topological space $X$, the very symmetric product $X<m>$ introduced in [R1] parametrises nonempty subsets of $X$ of cardinality $\leq m$. We now introduce an analogous space parametrising subsets with a distinguished subsubset. Define

$$
X<m, i>=\{(S, T): T \subset S\} \subset X<m+i>\times X<i>,
$$

with the induced topology as (closed) subset; thus $X<m, i>$ is just the graph of the tautological or incidence correspondence between $X<m+i>$ and $X<i>$. Note the natural continuous surjective map

$$
\begin{gathered}
\pi_{m, i}: X<m>\times X<i>\rightarrow X<m, i> \\
\left(S^{\prime}, S^{\prime \prime}\right) \longmapsto\left(S^{\prime} \cup S^{\prime \prime}, S^{\prime \prime}\right) .
\end{gathered}
$$

For $i=1$ is easy to see that via this map $X<m, 1>$ may be identified topologically with the quotient of $X<m>\times X$ by the relation identifying $\left(S^{\prime}, x\right) \in X<m-1>$ 
$\times X \subset X<m>\times X$ with $\pi_{m, 1}\left(S^{\prime}, x\right)=\left(S^{\prime} \cup\{x\}, x\right) \in X<m-1,1>\subset X<$ $m>\times X$

For sheaves $A, B$ on $X$-say of modules over some ring which will typically be $\mathbb{C}$-we (abusively) denote by $\lambda^{i} A \otimes B$, as sheaf on $X<i, 1>$, the direct image $\pi_{i, 1 *}\left(\lambda^{i} A \otimes B\right)$.

Now let $g$ be a sheaf of Lie algebras on $X$. In [R1] we associated to $g$ a Jacobi complex $J_{m}(g)$ on $X<m>$, an OS structure on $V^{m}(g)=\mathbb{H}^{0}\left(J_{m}(g)\right)$, and consequently a (commutative associative) artin local algebra structure on $R_{m}(g)=$ $\mathbb{C} \oplus \mathbb{H}^{0}\left(J_{m}(g)\right)^{*}$. Note that this construction carries over essentially verbatim to the case where $g$ is a differential graded Lie algebra (DGLA) sheaf ( i.e. a 'Lie object' is the category of complexes of sheaves on $X$-as opposed to ordinary sheaves); in the DGLA case $J_{m}$ becomes a double complex, but we shall generally identify it and other multiple complexes with the associated simple complex. See the Appendix for an interpretation of $R_{m}(g)$ in this case.

Now let $E$ be a $g$-module. We shall associate to $E$ a complex on $X<m, 1>$, called the modular Jacobi complex and denoted by $J_{m}(g, E)$. The terms are defined by $J_{m}^{i}(g, E)=\lambda^{i}(g) \otimes E$ on $X<i, 1>\subset X<m, 1>, 0 \leq i \leq m$ (where natually $X<0,1>$ is the diagonal $X \subset X \times X=X<1>\times X)$, and the differential $\partial_{i}: \lambda^{i}(g) \otimes E \rightarrow \lambda^{i-1}(g) \otimes E$ is given by the standard formula from Lie algebra homology:

$$
\begin{aligned}
\partial_{i}\left(v_{1} \times \cdots \times v_{i} \times e\right)= & \frac{1}{i !} \sum_{\sigma \in S_{i}}(\operatorname{sgn} \sigma)\left[v_{\sigma(j)}, v_{\sigma(k)}\right] v_{\sigma(1)} \times \cdots \times \hat{v}_{\sigma(j)} \times \cdots \times \hat{v}_{\sigma(k)} \times \cdots \times v_{\sigma(i)} \times e \\
& +\frac{1}{i} \sum_{j=1}^{i}(-1)^{j} v_{1} \times \cdots \times \hat{v}_{j} \times \cdots \times v_{i} \times v_{j}(e) .
\end{aligned}
$$


Now we have a natural map

$$
\begin{gathered}
\phi: X<m>\times X<m-1,1>\rightarrow<2 m, 1> \\
\left(S,\left(S^{\prime}, x\right)\right) \longmapsto\left(S \cup S^{\prime}, x\right)
\end{gathered}
$$

and an evident map of complexes

$$
\left(J_{m} / J_{1}\right)(g, E) \rightarrow \phi_{*}\left(J_{m}(g) \otimes J_{m-1}(g, E)\right)
$$

given by the natural sheaf maps, analogous to the map $\wedge^{i}(V) \rightarrow \oplus\left(\wedge^{i-k}(V) \otimes\right.$ $\left.\wedge^{k}(V)\right)$ for a vector space $V$

$$
\lambda^{i}(g) \otimes E \rightarrow \underset{k=0}{\oplus} \lambda^{i-k}(g) \otimes\left(\lambda^{k}(g) \otimes E\right)
$$

( it is easy to check that this is compatible with differentials). Now let $p$ : $X<m, 1>\rightarrow X$ be the natural map. Then by the above the sheaf $V^{m}(E):=$ $\mathbb{R}^{0} p_{*}\left(J_{m}(g, E)\right)$ on $X$ is endowed with an MOS structure with respect to the (constant) OS structure $\mathbb{H}^{0}\left(J_{m}(g)\right)$, whence a sheaf of $R_{m}(g)$-modules $C^{m}\left(V^{m}(E)\right)$ which we denote by $M_{m}(g, E)$. Note that the assignment $E \rightarrow M_{m}(g, E)$ is a covariant functor from $g$-modules to $R_{m}(g)$-modules.

\section{Universal variation of Hodge structure}

Let $\mathrm{X}$ be a compact complex manifold with tangent sheaf $T$ so that $H^{0}(T)=0$. In [R1] we constructed the universal formal deformation

$$
\hat{X}^{u} / \hat{R}^{u}=\lim _{m} X_{m}^{u} / R_{m}^{u},
$$

where $R_{m}^{u}=R_{m}(T)$ is the algebra associated to the OS structure $\mathbb{H}^{0}\left(J_{m}(T)\right)$. More generally if $g$ is a sheaf of $\mathbb{C}$-Lie algebra over a topological space $X$ with $H^{0}(g)=$ Oand $E$ is a sheaf of faithful $g$-modules, we have constructed the universal $m$-th 
order $g$-deformation $E_{m}$ of $E$ over $R_{m}(g)$, the algebra associated to $\mathbb{H}^{0}\left(J_{m}(g)\right)$. A first point to be made is the the MOS viewpoint permits a more 'conceptual' interpretation of this construction (I am grateful to the referee for his insistence that this be explained in detail).

Theorem 3.1. $E_{m}$ is canonically isomorphic to $M_{m}(g, E)$.

Proof. Let $(E, \partial)$ be a soft resolution of $E$ and $(g \cdot \delta)$ be a soft resolution of $g$ where $g$ is a dgla acting on $E$. compatibly with the $g$-action on $E$. Applying suitable Schur functors as in $[\mathrm{FH}], g$ induces a soft resolution of $\lambda^{i}(g)$ which we denote by $\left(g_{-i}^{\cdot}, \delta_{-i}\right)$, whence also a soft resolution of $J_{m}(g, E)$, which may be used to compute $p_{*}\left(J_{m}(g, E)\right)$, yielding a complex $(K \cdot d \cdot)$ on $X$ where

$$
K^{r}=\sum_{i, j} \Gamma\left(g_{i+j+r}^{i}\right) \otimes E^{i}, i \geq-m
$$

Now note that, because $H^{0}(g)=0$, we have $H^{j}\left(\lambda^{i}(g)\right)=0, j<i$, hence $K$. is acyclic in negative degrees. More precisely, we may 'cancel off' the negative part of $K$ step-by-step as follows. First $d^{-m}$ is a map

$$
\Gamma\left(g_{-m}^{0}\right) \otimes E^{0} \rightarrow \Gamma\left(g_{-m}^{1}\right) \otimes E^{0} \oplus \Gamma\left(g_{-m}^{0}\right) \otimes E^{1} \oplus \Gamma\left(g_{-m+1}^{0}\right) \otimes E^{0}
$$

whose first component $\delta_{-m}^{0} \otimes i d_{E^{0}}$ is already injective. Consequently $K \cdot$ is quasiisomorphic to a complex $K_{(1)}$ where

$$
K_{(1)}^{-m}=0, K_{(1)}^{-m+1}=\left(\Gamma\left(g_{-m}^{1}\right) / B^{1}\left(g_{-m}^{\cdot}\right)\right) \otimes E^{0} \oplus \Gamma\left(g_{-m}^{0}\right) \otimes E^{1} \oplus \Gamma\left(g_{-m+1}^{0}\right) \otimes E^{0},
$$

and $K_{(1)}^{i}=K^{i}, i>-m+1$. Here $B$ denotes coboundaries. To be precise $\Gamma\left(g_{-m}^{1}\right) / B^{1}\left(g_{-m}^{\cdot}\right)$ is to be thought of as a subspace of $\Gamma\left(g_{-m}^{1}\right)$ complementary to $B^{1}\left(g_{-m}^{\cdot}\right)$. Now the map induced by $\delta_{-m}^{1} \otimes i d_{E^{0}}$ on $\left(\Gamma\left(g_{-m}^{0}\right) / B^{1}\left(g_{-m}^{\cdot}\right)\right) \otimes E^{0}$ is still injective if $m \geq 2$ 
( again thanks to $H^{0}(g)=0$ ), as are $\delta_{-m}^{0} \otimes i d_{E^{1}}$ and $\delta_{-m+1}^{0} \otimes i d E^{0}$, hence $K_{(1)}$ is in turn quasi-isomorphic to a complex $K_{(2)}$ in degrees $\geq-m+2$ with

$$
\begin{gathered}
K_{(2)}^{-m+2}=\left(\Gamma\left(g_{-m}^{2}\right) / B^{2}\left(g_{-m}^{\cdot}\right)\right) \otimes E^{0} \oplus\left(\Gamma\left(g_{-m}^{1}\right) / B^{1}\left(G_{-m}^{\cdot}\right)\right) \otimes E^{1} \\
\oplus\left(\Gamma\left(g_{-m+1}^{1}\right) / B^{1}\left(g_{-m+1}^{\cdot}\right)\right) \otimes E^{0} \oplus \ldots
\end{gathered}
$$

Continuing in this manner, we obtain after $m$ steps a complex $\left(K_{(m)}, d_{(m)}\right)$ in nonnegative degrees where $K_{(m)}^{0}$ is of the form $\sum_{i \geq 0} G_{i} \otimes E^{i}$ for certain vector spaces $G^{i}$. Now it is easy to see as above that for $i>0$ the map $\delta \otimes i d_{E^{i}}$ which is a component of $d_{(m)}^{0}$ is injective. For $i=0, G_{0}$ will be a certain quotient of $\sum_{0}^{m} \operatorname{sym}^{j}\left(\Gamma\left(g^{1}\right)\right.$ and among the components of $d_{m}^{0}$ on $G^{0} \otimes E^{0}$, one lands in $\Gamma\left(g^{2}\right) \otimes \sum_{1}^{m} \operatorname{sym}^{j-1}\left(\Gamma\left(g^{1}\right)\right) \otimes E^{0}\left(\right.$ map induced by $(\delta$ plus graded bracket $\left.) \otimes i d_{E^{0}}\right)$. By definition, the kernel of this map is precisely $V_{0}^{m} \otimes E^{0}=\left(\mathbb{C} \oplus \mathbb{H}^{0}\left(J_{m}(g)\right)\right) \otimes E^{0}$ and consequently $K_{(m)}$ is quasi isomorphic to a complex $K_{(m+1)}$ in nonnegative degrees with $K_{(m+1)}^{0}=V_{0}^{m} \otimes E^{0}$ and, say, $K_{(m+1)}^{1}=\sum H^{i} \otimes E^{i}$ for certain vector spaces $H^{i}$. By similar considerations $K_{(m+1)}$ is quasi- isomorphic to a complex $\left(L \cdot \Delta^{\cdot}\right)$ which starts

$$
V_{0}^{m} \otimes E^{0} \rightarrow V_{0}^{m} \otimes E^{1} \oplus L_{0}^{1} \rightarrow \ldots
$$

and where $\Delta^{0}$ which is induced by $i d \otimes \partial^{0}+$ (map induced by $g$-action) clearly goes into $V_{0}^{m} \otimes E^{1}$. Thus $\mathbb{R}^{0} p_{*} J_{m}(g, E)$ is simply given by the kernel of $\Delta^{0}$. In light of Proposition 1.1 and its proof, $M_{m}(g, E)$ coincides with the kernel of a map

$$
R_{m}(g) \otimes E^{0} \rightarrow R_{m}(g) \otimes E^{1}
$$

given by $i d \otimes \partial^{0}+($ map induced by action), which is precisely the definition of $E_{m}$ 
For later use we record a corollary of the construction (which follows easily from the fact that cocycles are locally coboundaries)

Corollary 3.2. $V^{m}(E)$ is locally isomorphic to $V_{0}^{m} \otimes E$.

Our purpose now is to extend this construction to the De Rham complex of $X$ and consequently to obtain, for $X$ Kählerian, a construction of the universal variation of Hodge structure associated to $X$, which is a (cohomology) vector bundle over $\operatorname{Spec}\left(\hat{R}^{u}\right)$ together with a (Hodge) filtration and a ( Gauss-Manin) trivialization or flat structure.

Consider the De Rham complex of X

$$
\Omega: \mathcal{O}_{X} \stackrel{d}{\rightarrow} \Omega_{X}^{1} \rightarrow \cdots \rightarrow \Omega_{X}^{n}
$$

As is well known, $T$ acts on this via Lie derivative $v \times \omega \longmapsto L_{v}(\omega)$, an action which commutes with exterior derivative and is a derivation with respect to wedge products. Consequently, we have an associated modular Jacobi (bi)complex $J_{m}(T, \Omega)$ on $X<m, 1>$ which we call the Jacobi-De Rham complex of $X$. This gives rise to a $V^{m}$ - compatible MOS structure $\mathbb{R}^{0} p_{*} J_{m}(T, \Omega)$ (i.e a complex of sheaves of such), whence a complex of $R_{m}^{u}=R_{m}(T)$-modules

$$
\Omega_{m}:=M_{m}\left(T, \Omega^{*}: M_{m}(T, \mathcal{O})=\mathcal{O}_{m} \rightarrow M_{m}\left(T, \Omega^{1}\right) \rightarrow \cdots\right.
$$

As remarked above, $\mathcal{O}_{m}$ coincide with $\mathcal{O}_{X_{m}}$, the structure sheaf of the $m$-universal deformation $X_{m}^{u} / R_{m}^{u}$. This identification may be extended as follows.

Theorem 3.3. In the above situation, $\Omega_{m}$ is canonically isomorphic to the relative De Rham complex $\Omega_{X_{m} / R_{m}}$. 
Proof. For $\cdot=0$ we're OK, so consider the case $\cdot=1$. The $T$-linear derivation $d: \mathcal{O} \rightarrow \Omega^{1}$ gives rise by functoriality to an $R_{m}^{u}$-linear derivation $\mathcal{O}_{m} \rightarrow \Omega_{m}^{1}$, whence an $\mathcal{O}_{m}$-linear map

$$
\phi_{m}^{1}: \Omega_{X_{m} / R_{m}}^{1} \rightarrow \Omega_{m}^{1}
$$

Clearly $\phi_{m}^{1} \otimes_{R_{m}} \mathbb{C}=\phi_{0}^{1}$ is the identity. Moreover it is easy to see that both sheaves are locally $\mathcal{O}_{m}$-free: for $\Omega_{X_{m} / R_{m}}^{1}$ this is obvious and for $\Omega_{m}^{1}$ it follows from the fact that it is $R_{m}$-flat by construction (indeed a local basis for $\Omega^{1}$ yields a map $n \mathcal{O}_{m} \rightarrow \Omega_{m}^{1}$ which is clearly surjective and whose kernel yields 0 when tensored with $\mathbb{C}$, hence is 0 by Nakayama). Consequently, a suitable $n \times n$ matrix representing $\phi_{m}^{1}$ will have the form $I_{n}+$ (nilpotent), hence is invertible, so $\phi_{m}^{1}$ is an isomorphism. Now in general, for $\cdot=i$ the derivation property of the Lie derivative action of $\mathrm{T}$ on $\Omega_{X}$ makes $\Omega_{m}$ an algebra under wedge products, whence an $\mathcal{O}_{m}$-linear map

$$
\phi_{m}^{i}=\wedge^{i}\left(\phi_{m}^{1}\right): \wedge_{\mathcal{O}_{m}}^{i}\left(\Omega_{X_{m} / R_{m}}^{1}\right) \rightarrow \Omega_{m}^{i}
$$

As both sheaves are locally $\mathcal{O}_{m}$-free and $\phi_{o}^{i}$ is the identity, it follows as above that $\phi_{m}^{i}$ is an isomorphism. Compatibility of $\phi_{m}$ with $d$-i.e, $d \circ \phi_{m}^{i}=\phi_{m}^{i+1} \circ d$ follows easily from the fact that this holds for $i=0$ (by construction) and the multiplicativity of $\phi_{m}$. This completes the proof.

Given Theorem 3.2 it is clear in principle that the De Rham cohomology of $X_{m} / R_{m}$ is readable from the cohomology of the Jacobi-DeRham complex and we shall now make this explicit. Consider the free $R_{m}$-module

$$
H_{D R}^{r}\left(X_{m} / R_{m}\right)=\mathbb{H}^{r}\left(X, \Omega_{X_{m} / R_{m}}\right) .
$$


This is endowed with a Hodge filtration $F$, which is induced by the stupid filtration on the complex $\Omega_{X_{m} / R_{m}}$, as well as a (Gauss-Manin) isomorphism $H_{D R}^{r}\left(X_{m} / R_{m}\right) \simeq$ $H^{r}\left(X, R_{m}\right) \simeq H^{r}(X, \mathbb{C}) \otimes R_{m}$ induced by the (quasi-isomorphic) inclusion $R_{m} \rightarrow$ $\Omega_{X_{m} / R_{m}}$. When $X$ is Kählerian we have by Deligne [D] that $F^{i} / F^{i+1} \cong H^{n-i}\left(\Omega_{X_{m} / R_{m}}^{i}\right)$, a free $R_{m}$-module. The filtered module $\left(H_{D R}^{r}\left(X_{m} / R_{m}\right), F^{*}\right)$ may be called the $m$ universal variation of Hodge structure ( $m$-UVHS) associated to $X$. Our goal is to give an explicit formula for it, together with the Gauss-Manin isomorphism, in terms of $X$ itself.

Theorem 3.4. Let $X_{m} / R_{m}$ be the m-universal deformation of a compact kähler manifold $X$ with $H^{0}(T)=0$. Then: (i) the Leray spectral sequence

$$
E_{2}^{p, q}=\mathbb{H}^{p}\left(X, R^{q} p_{*} J_{m}\left(T, \Omega^{\cdot}\right)\right) \Rightarrow \mathbb{H}^{p+q}\left(X<m, 1>, J_{m}\left(T, \Omega^{*}\right)\right)
$$

degenerates at $E_{2}$;

(ii) we have $R_{m}$-linear, Hodge filtration-preserving isomorphisms

$$
\begin{aligned}
E_{2}^{r, 0}=E_{\infty}^{r, 0}=\mathbb{H}^{r}\left(X, B^{m} \Omega_{X_{m} / R_{m}}\right)=B^{m} \mathbb{H}^{r}\left(X, \Omega_{X_{m} / R_{m}}\right) & \\
\mathbb{H}^{r}\left(X, \Omega_{X_{m} / R_{m}}\right) & \cong C^{m} \mathbb{H}^{r}\left(X, \mathbb{R}^{0} p_{*} R_{m}(T, \Omega)\right) \\
& \subset C^{m} \mathbb{H}^{r}\left(X<m, 1>, J_{m}(T, \Omega)\right)
\end{aligned}
$$

(iii) the Jacobi-Hodge-De Rham spectral sequence

$$
E_{1}^{p, q}=\mathbb{H}^{p}\left(X, \mathbb{R}^{0} p_{*} J_{m}\left(T, \Omega^{q}\right)\right) \Rightarrow \mathbb{H}^{p+q}\left(X, \mathbb{R}^{0} p_{*} J_{m}\left(T, \Omega^{\cdot}\right)\right)
$$

degenerates at $E_{1}$;

(iv) the Gauss-Manin isomorphism

$$
G M_{m}: H_{D R}^{r}\left(X_{m} / R_{m}\right) \rightarrow H_{D R}^{r}(X) \otimes R_{m}
$$


is adjoint to a map $G M_{m}: B^{m} H_{D R}^{r}\left(X_{m} / R_{m}\right) \rightarrow H_{D R}^{r}(X) \otimes\left[\mathbb{C} \oplus \mathbb{H}^{0}\left(J_{m}(T)\right)\right]$ induced by a map of complexes

$$
M_{m}: J_{m}\left(T, \Omega^{*}\right) \rightarrow J_{m}\left(T, \Omega_{\text {triv }}\right)
$$

$\Omega_{\text {triv }}=\Omega$ with trivial T-action, which respects the Hodge filtration up to a shift of $m$, i.e. $M_{m}\left(F^{i}\right) \subset F^{i-m}$, hence $G M_{m}\left(F^{i}\right) \subset F^{i-m}$.

Proof. To begin with, the inclusion

$$
\mathbb{C}_{X} \rightarrow \Omega
$$

is, by the Poincaré lemma, a $T$-linear isomorphism (with the trivial action of $T$ on $\left.\mathbb{C}_{X}\right)$. Now recall the finite-to-one map

$$
\pi: X<m>\times X \rightarrow X<m, 1>
$$

(cf. section 2) and let $X$ be embedded diagonally $X \hookrightarrow X \times X=X<1>\times X \subset$ $X<m>\times X$. Then it follows that

$$
J_{m}\left(T, \Omega^{*}\right) \underset{q i s}{\sim} J_{m}(T, \mathbb{C}) \underset{q i s}{\sim} \pi_{*}\left(p_{1}^{*} J_{m}(T) \oplus p_{2}^{*} \mathbb{C}_{X}\right)
$$

This clearly implies the degeneration assertion of $(i)$. As for $(i i)$, we have proven in Theorem 3.1 that

$$
\Omega_{X_{m} / R_{m}} \simeq C^{m}\left(\mathbb{R}^{0} p_{*} J_{m}\left(T, \Omega^{\cdot}\right)\right)
$$

whence a map

$$
\mathbb{R}^{0} p_{*} J_{m}\left(T, \Omega^{*}\right) \rightarrow B^{m} \Omega_{X_{m} / R_{m}} \simeq B^{m} C^{m} \mathbb{R}^{0} p_{*} J_{m}\left(T, \Omega^{\cdot}\right)
$$


It is easy to see that this is an isomorphism for $m=0$, and both sides are $V^{m}$-coflat, locally a sum of copies of $V_{0}^{m} \otimes \mathcal{O}_{X}$, hence this is an isomorphism. On the other hand $\Omega_{X_{m} / R_{m}}$ being $R_{m}$-flat we have

$$
\mathbb{H}^{r}\left(X, \mathbb{R}^{0} p_{*} J_{m}\left(T, \Omega^{*}\right)\right)=\mathbb{H}^{r}\left(X, B^{m} \Omega_{X_{m} / R_{m}}\right)=B^{m} \mathbb{H}^{r}\left(X, \Omega_{X_{m} / R_{m}}\right) .
$$

This proves (3.1). Applying $C^{m}$ to both side and using $R_{m}$-freeness of $\mathbb{H}^{r}\left(X, \Omega_{X_{m} / R_{m}}\right)$, (3.2) follows too. Given (ii), (iii) follows easily from degeneration of the usual (relative) Hodge-De Rham spectral sequence

$$
E_{1}^{p, q}=H^{q}\left(X, \Omega_{X_{m} / R_{m}}^{p}\right) \Rightarrow H_{D R}^{p+q}\left(X_{m} / R_{m}\right)
$$

Finally for $(i v)$-which is really the main point-we shall construct an explicit isomorphism based on interior multiplication. For local vector fields $v_{1}, \cdots, v_{k}$ we let

$$
i_{v_{1} \cdots v_{k}}: \Omega^{i} \rightarrow \Omega^{i-k}
$$

denote interior multiplication by $v_{1} \times \cdots \times v_{k}$ ( which coincides with $\left.i_{v_{1}} \circ \cdots \circ i_{v_{k}}\right)$.

Recall Cartan's formula for the Lie derivative action of $T$ on $\Omega$ :

$$
L_{v}(\omega)=i_{v}(d(\omega))+d\left(i_{v}(\omega)\right) .
$$

Now let us define a map $M$, preserving total degree

$$
\begin{gathered}
M=M^{\cdots}: J_{m}\left(T, \Omega^{*}\right) \rightarrow J_{m}\left(T, \Omega_{\text {triv }}\right) \\
M^{i, j, k} \quad: \lambda^{j} T \otimes \Omega^{i} \quad \rightarrow \lambda^{j-k} T \otimes \Omega^{i-k}, k \geq 0, \\
M^{i, j, k}\left(v_{1} \times \cdots \times v_{j} \times \omega\right)= \pm \sum(-1)^{r_{1}+\cdots+r_{k}} v_{1} \times \cdots \times \hat{v}_{r_{1}} \times \cdots \times \hat{v}_{r_{k}} \times \cdots \times v_{j} \times i_{v_{r_{1}} \cdots v_{r_{k}}}(\omega) .
\end{gathered}
$$


In other words $M$ is the map whose restriction on $\lambda^{j} T \otimes \Omega^{i}$ is given by $\oplus_{k \geq 0} M^{i, j, k}$; note that each $M^{i, j, 0}$ is the 'identity'. The Cartan formula shows that $M$ is a morphism of complexes, whence a map

$$
\begin{aligned}
{ }^{t} G M_{m}: \mathbb{H}^{r}\left(X, \mathbb{R}^{0} p_{*} J_{m}\left(T, \Omega^{\cdot}\right)\right) & \rightarrow \mathbb{H}^{r}\left(X, \mathbb{R}^{0} p_{*} J_{m}\left(T, \Omega_{\text {triv }}\right)\right. \\
& =\mathbb{H}^{r}\left(\Omega_{X}\right) \otimes\left[\mathbb{C} \oplus \mathbb{H}^{0}\left(J_{m}(T)\right)\right]
\end{aligned}
$$

In view of the commutative triangle

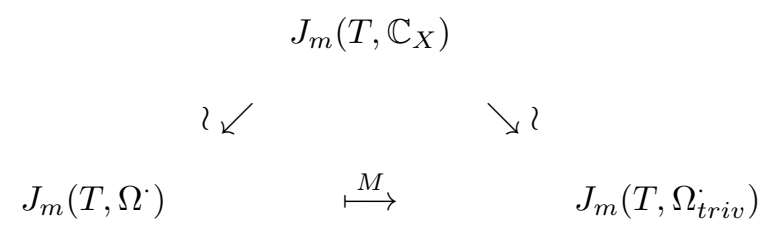

${ }^{t} G M_{m}$ is an isomorphism, and it is obvious that it decreases Hodge level by at most $m$. Applying the $C^{m}$ functor now suffices to conclude. Note that by construction the inverse of our GM isomorphism is induced by the quasi-isomorphism $R_{m} \rightarrow$ $\Omega_{X_{m} / R_{m}}$; the same is obviously true of the 'usual' Gauss-Manin in any way it is defined (e.g. $[\mathrm{K}]$ ). Thus our GM coincides with the usual.

The following discussion concerning cohomology and obstructions was suggested by some recent work of Clemens $[\mathrm{C}]$. Consider the exact sequence

$$
0 \rightarrow m_{m}^{m} \otimes \Omega^{p} \rightarrow \Omega_{m}^{p} \rightarrow \Omega_{m-1}^{p} \rightarrow 0
$$

By [D] (assuming $X$ Kählerian) this induces an exact sequence

$$
0 \rightarrow m_{m}^{m} \otimes H^{q}\left(\Omega^{p}\right) \rightarrow H^{q}\left(\Omega_{m}^{p}\right) \rightarrow H^{q}\left(\Omega_{m-1}^{p}\right) \rightarrow 0
$$

i.e. the coboundary map $\partial_{m}: H^{q}\left(\Omega_{m-1}^{p}\right) \rightarrow m_{m}^{m} \otimes H^{q+1}\left(\Omega^{p}\right)$ vanishes, and $H^{q}\left(\Omega_{m}^{p}\right)$ is $R_{m}^{u}$-free. It is easy to see that the vanishing of $\partial_{m-1}$ implies a priori that $\partial_{m}$ 
factors through a map $H^{q}\left(\Omega^{p}\right) \rightarrow m_{m}^{m} \otimes H^{q+1}\left(\Omega^{p}\right)$. 'Dually', let $K^{m} \subset \operatorname{sym}^{m} H^{1}(T)$ be the kernel of the $m$-th order obstruction map $o b_{m}: \operatorname{sym}^{m} H^{1}(T) \rightarrow \mathbb{H}^{1}\left(J_{m-1}(T)\right.$ (cf. [R2],2.3). Alternatively, $K^{m}$ may also be defined inductively as the kernel of an obstruction map $o b_{m}: K^{m-1} \cdot H^{1}(T) \rightarrow H^{2}(T)$, where $K^{m-1} \cdot H^{1}(T)$ denotes the intersection of $\operatorname{sym}^{m} H^{1}(T)$ and $K^{m-1} \otimes H^{1}(T)$ in $\otimes^{m} H^{1}(T)$. Then the natural map

$$
K^{m} \otimes H^{q}\left(\Omega^{p}\right) \rightarrow H^{q+1}\left(\Omega^{p}\right)
$$

which represents the obstruction to lifting $(p, q)$-cohomology over the universal $m$ th order deformation, vanishes. Equivalently, the tautological map $J_{m}\left(T, \Omega^{p}\right) \rightarrow$ $\lambda^{m}(T) \otimes \Omega^{p}[m]$ induces a map $V^{m}\left(\Omega^{p}\right) \rightarrow \operatorname{sym}^{m} H^{1}(T) \otimes \Omega^{p}$ whose image coincides with $K^{m} \otimes \Omega^{p}$ (cf. Corollary 3.2). Then the induced map on cohomology

$$
H^{q}\left(V^{m}\left(\Omega^{p}\right)\right) \rightarrow K^{m} \otimes H^{q}\left(\Omega^{p}\right)
$$

is surjective, i.e the coboundary or obstruction map

$$
K^{m} \otimes H^{q}\left(\Omega^{p}\right) \rightarrow H^{q+1}\left(V^{m-1}\left(\Omega^{p}\right)\right)
$$

vanishes (and moreover $H^{q}\left(V^{m}\left(\Omega^{p}\right)\right)$ is cofree). This follows from [D] together with a trivial 'Universal coefficient theorem' which says that $H^{q}\left(B^{m}\left(\Omega_{m}^{p}\right)\right)=$ $B^{m}\left(H^{q}\left(\Omega_{m}^{p}\right)\right)$ provided $H^{q-1}\left(\Omega_{m}^{p}\right)$ is free; alternatively one can simply mimic Deligne's semicontinuity argument for $V^{m}\left(\Omega^{p}\right)$ in place of $\Omega_{m}^{p}$.

Now recall the calculus fact (already used in [R3]):

$$
i([x, y])(\omega)=L_{x}(i(y) \omega)-L_{y}(i(x) \omega)-d(i(x \wedge y) \omega)+i(x \wedge y)(d \omega) .(*)
$$

Define a map

$$
i: J_{m}(T) \otimes \Omega^{p} \rightarrow J_{m-1}\left(T, \Omega^{p-1}\right)[1]
$$


by the formula

$$
\left(v_{1}, \ldots, v_{k}, \omega\right) \mapsto \sum(-1)^{j}\left(v_{1}, \ldots \hat{v}_{j}, \ldots, v_{k}, i\left(v_{j}\right) \omega\right)
$$

Then $\left(^{*}\right)$ shows, assuming $X$ Kähler, that $i$ is a 'weak' morphism of complexes, in the sense that the appropriate diagrams commute in the derived category. This is good enough to induce a map on cohomology

$$
V^{m} \otimes H^{q}\left(\Omega^{p}\right) \rightarrow H^{q}\left(V^{m-1}\left(\Omega^{p-1}\right)\right)
$$

and similarly for $m-1$, hence a commutative diagram

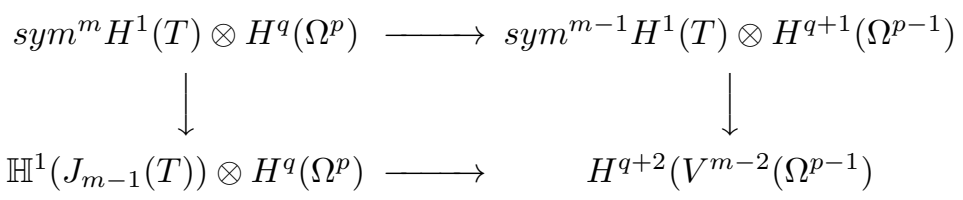

which induces

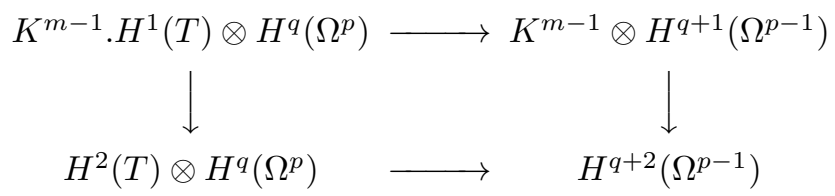

As we have seen, the right vertical map vanishes, and we conclude the following which was first proven by Clemens $[\mathrm{C}]$ by another method:

Corollary 3.5. (Clemens) For $X$ Kählerian, the map $H^{2}(T) \rightarrow \operatorname{Hom}\left(H^{q}\left(\Omega^{p}\right), H^{q+2}\left(\Omega^{p-1}\right)\right)$ vanishes on the image of the $m$-th order obstruction map in $H^{2}(T)$, for all $m$.

It is instructive to rewrite some of the conclusion of Theorem 3.4 in traditional geometric language. Thus $H_{m}^{r}=H_{D R}^{r}\left(X_{m} / R_{m}\right)$ may be viewed as a geometric 
vector bundle-viz. $\operatorname{Spec}\left(\operatorname{Sym}\left(H_{m}^{r *}\right)\right)$-over $\operatorname{Spec}\left(R_{m}\right)=: U_{m}$ and the Gauss-Manin isomorphism yields a commutative triangle:

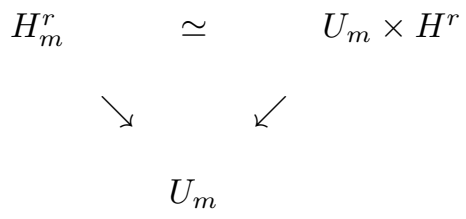

where $H^{r}=H_{0}^{r}=H_{D R}^{r}(X)$. The geometric vector bundles $H_{m}^{r}$ and $U_{m} \times H^{r}$ correspond to OS structures over $V_{m}$ which are the 'symmetric coalgebra' over $V^{m}$ (see Remark 1.2) on the MOS structures $H^{r}\left(\mathbb{R}^{0} p_{*} J_{m}\left(T, \Omega^{\cdot}\right)\right)$ and $H^{r}\left(\mathbb{R}^{0} p_{*} J_{m}\left(T, \Omega_{\text {triv }}\right)\right)$, respectively, the latter being evidently isomorphic to $V^{m} \otimes S^{\cdot}(H)$, where $S^{\cdot}(H)$, the symmmetric coalgebra (= algebra) on $H$ over $\mathbb{C}$ is endowed with the evident OS structure (e.g. via its duality with $S^{\cdot}\left(H^{*}\right)$ ). Now the Hodge subbundle $F_{m}^{i} \subset H_{m}^{r}$, together with the trivialization (3.4), give rise to a morphism to a Grassmannian

$$
p_{m}: S_{m} \rightarrow \operatorname{Grass}\left(\operatorname{dim} F_{0}^{i}, H^{r}\right),
$$

which is none other than the $m$-th order germ of the period map associated to $\left(X, H^{r}, T^{i}\right)$. Thus Theorem 3.2 yields, in principle, an explicit formula for $p_{m}$. In the next section we shall make this concrete in the case of Calabi-Yau manifolds.

\section{The Calabi-Yau case}

In this section we fix a Calabi-Yau manifold $X$, i.e. an $n$-dimensional compact Kähler manifold admitting a nowhere-vanishing $n$-form $\Phi$ (which is then unique up to a constant), and such that $H^{0}(T)=0$. We call such a pair $(X, \Phi)$ a measured Calabi-Yau manifold (MCYM). A (local) isomorphism $\mathrm{f}$ between MCYM's $(X, \Phi),\left(X^{\prime}, \Phi^{\prime}\right)$ is supposed to preserve the $n$-form, i.e. $f^{*} \Phi^{\prime}=\Phi$. Thus the Lie algebra sheaf of infinitesimal automorphisms of a MCYM $(X, \Phi)$ may be identified 
as the subset $\hat{T} \subset T$ of divergence-free vector fields $v$, i.e. those with $L_{v}(\Phi)=0$. The inclusion of Lie algebras $\hat{T} \subset T$ corresponds to an inclusion of rings

$$
R_{m}=R_{m}(T) \subset \hat{R}_{m}=R_{m}(\hat{T})
$$

Indeed it is easy to see that $\hat{R}_{m}=R_{m}[t] /(\underline{m}, t)^{m+1}$. The universal deformation over $\hat{R}_{m}$ is just $X_{m}^{n} \times_{R_{m}} \hat{R}_{m}$. The advantage of $\hat{R}_{m}$ is that the pullback of the cohomology bundle $H_{m}^{n}=H_{D R}^{n}\left(X_{m}^{u} / R_{m}\right)$ over $\hat{U}_{m}=\operatorname{Spec}\left(\hat{R}_{m}\right)$ admits a 'tautological' section: namely that corresponding to the map

$$
J_{m}(\Phi): J_{m}(\hat{T}, \mathbb{C}[-n]) \rightarrow J_{m}(\hat{T}, \Omega)
$$

induced by the $\hat{T}$-linear (!) map $\mathbb{C}[-n] \stackrel{\Phi}{\rightarrow} \Omega$. To be precise, there is an inclusion (of a direct summand)

$$
\pi_{*} p_{1}^{*} J_{m}(\hat{T})[-n] \hookrightarrow J_{m}(\hat{T}, \mathbb{C}[-n])
$$

(indeed $\pi_{*} p_{1}^{*} J_{m}(\hat{T})[-n]$ is identical with the part of $J_{m}(h a t T, \mathbb{C}[-n])$ in negative degrees and this part forms a sub, as well as quotient, complex because the differential in degree -1, given by the action, vanishes). This inclusion induces

$$
[\Phi]: \mathbb{H}^{0}\left(J_{m}(\hat{T})\right) \rightarrow \mathbb{H}^{n}\left(\mathbb{R}^{0} p_{*} J_{m}\left(\hat{T}, \Omega^{\cdot}\right)\right)
$$

which corresponds to $\Phi$ as a cross-section of the geometric vector bundle corresponding to $H_{m}^{n} \otimes \hat{R}_{m}$. On the ring level this corresponds to the $\hat{R}_{m}$-algebra homomorphism

$$
S_{\hat{R}_{m}}\left(\left(H_{m}^{n}\right)^{*} \otimes \hat{R}_{m}\right) \rightarrow \hat{R}_{m}
$$

given by the composite (where $S \cdot=S_{\mathbb{C}}$ )

$$
S \cdot\left(\left(H_{m}^{n}\right)^{*}\right) \rightarrow S \cdot\left(\hat{R}_{m}\right) \rightarrow \hat{R}_{m}
$$


where the first map is $S \cdot[\Phi]^{*}$ and the second is $\hat{R}_{m}$-multiplication. Now we may follow this by the map induced by the Gauss- Manin isomorphism $H_{m}^{n} \simeq H^{n} \otimes R_{m}$ (3.4), then project to the $H^{n}$ factor. The map thus obtained

$$
\hat{p}_{m}: \hat{S}_{m} \rightarrow H^{n}=: H
$$

is the $m$-th order period map associated to the $\operatorname{MCYM}(X, \Phi)$. This is related to the usual period map $p_{m}$ by the diagram

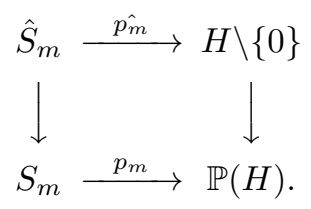

Now $\hat{p}_{m}$ corresponds to a homomorphism $\hat{p}_{m}^{*}: S \cdot\left(H^{*}\right) \rightarrow \hat{R}_{m}$, which is obviously determined by its restriction $\hat{p}_{m}^{1 *}$ on $H^{*}$, the linear functions on $H$. Tracing through the construction of the Gauss-Manin isomorphism in Theorem 3.2, we conclude the following formula for the dual $\hat{p}_{m}^{1}$ of $\hat{p}_{m}^{1 *}$ :

Theorem 4.1. The map $\hat{p}_{m}^{1}: \mathbb{H}^{0}\left(J_{m}(\hat{T})\right) \rightarrow H_{D R}^{n}(X)$ is given by $\mathbb{H}^{0}\left(j_{m}\right)$ where $j_{m}: J_{m}(\hat{T}) \rightarrow \Omega[n]$ is defined by $j_{m}\left(v_{1} \times \cdots \times v_{k}\right)=i_{v_{1} \cdots v_{k}}(\Phi)$.

Proof. Our map is given as the composite of three maps. First, embedding $J_{m}(\hat{T})$ in the negative-degree portion of the $n$-th column (viz. $J_{m}\left(\hat{T}, \Omega^{n}\right)$ ) of the double complex $J_{m}(\hat{T}, \Omega \cdot)$ through multiplication by $\Phi$. Second, applying our explicit Gauss-Manin (interior multiplication) operator $M$ constructed in the proof of Theorem 3.2. Third, projecting to the zeroth row, which is just given by $\Omega$. From our formula for $M(3.3)$, is is plain that this map is given by $\mathbb{H}^{0}\left(j_{m}\right)$.

We shall now use this description of the period map to describe a set of defining equations for its image (Schottky relations). For convenience, set $\hat{T}^{m}=\mathbb{H}^{0}\left(J_{m}(\hat{T})\right)$, 
$\underline{m}_{m}=\hat{T}^{m *}$, the maximal ideal of $\hat{R}_{m}$. To mirror the adic filtration on $\hat{R}_{m}$ we define a slight modification $\hat{F}$ of the Hodge filtration on $H$ (which we identify with its dual $H^{*}$ by Poincaré duality) by

$$
\begin{aligned}
\hat{F}^{i} H & =\quad H \quad i=1 \\
& =F^{i} H \quad i \neq 1 .
\end{aligned}
$$

$\hat{F}$ naturally induces a filtration on the symmetric algebra $S(H),(=$ unique filtration such that the level of $a b=($ level of $a)+($ level of $b))$ and we denote by $S_{m}$ its $m$-th quotient $S(H) / \hat{F}^{m+1} S(H)$. Note that e.g. by Theorem $4.1, \hat{p}_{m}^{1 *}$ on $H$ takes $\hat{F}$ into the adic filtration on $\hat{R}_{m}$. As $\hat{F}$ was extended 'multiplicatively' to $S \cdot(H), \hat{p}_{m}^{*}$ also takes $\hat{F}$ into the adic filtration, yielding a map $S_{m} \rightarrow \hat{R}_{m}$. It is also clear that $\hat{p}_{m}^{*}$ induces an isomorphism

$$
\operatorname{gr}_{\hat{F}}^{1} S(H)=H / F^{2} H \simeq g r^{1}\left(\hat{R}_{m}\right)=\hat{T}^{1 *}
$$

It follows firstly that $\hat{p}_{m}^{*}$ is surjective('local Torelli') but also, more significantly that for any $a \in \hat{F}^{i} H$ we may choose $b \in \oplus_{j=i}^{n} S^{j} H$ so that

$$
\hat{p}_{n}^{*}(a)=\hat{p}_{n}^{*}(b) \in \underline{m}_{n}^{i} \subset \hat{R}_{n}
$$

Indeed writing $b=\sum_{i}^{n} b_{j}$ we may firstly choose $b_{i} \in S^{i}(H)$ by (4.0) so that $\hat{p}_{n}^{*}(a-$ $\left.b_{i}\right) \in \underline{m}^{i+1}$, then choose $b_{i+1} \in S^{i+1}(H)$ so that $\hat{p}_{n}^{*}\left(a-b_{i}-b_{i+1}\right) \in \underline{m}^{i+1}$, etc. We let $Y_{n}$ denote the set of elements $a-b$ of this kind as $a$ ranges over (a basis of ) $F^{i} H$, $i=2, \cdots, n$. These are our 'Schottky relations' of order $n$; they are essentially a refined version of the well-known Yukawa forms

$$
\eta^{i}: S^{i}\left(H^{n-1,1}\right) \rightarrow H^{n-i, i}
$$


in that $a$ defines a linear form on $H^{n-i, i}$ and $a \circ \eta^{i}$ is 'given' by (the degree- $i$ part of ) $b$ as linear form on $S^{i}\left(H^{n-1,1}\right)$. Next we define our Schottky relations of higher order. For each $m \geq n$ we define by induction a lift $Y_{m}$ of $Y_{n}$,

$$
Y_{m} \subset I_{m}=\operatorname{ker}\left(S_{m} \rightarrow \hat{R}_{m}\right)
$$

as follows. For any $y \in I_{m}$ let $y^{\prime} \in S_{m+1}$ be an arbitrary lift of $y$. Thus $\hat{p}_{m+1}^{*}\left(y^{\prime}\right) \in$ $\underline{\hat{\underline{h}}}_{m+1}^{m+1}$ may be expressed as a polynomial of degree $m+1$ in $\hat{T}^{1 *}$, which may be represented by an element $z \in S^{m+1}(H)$ and we clearly have $p_{m+1}^{*}(z)=p_{m+1}^{*}(y)$.

Now set

$$
\begin{gathered}
y^{\prime \prime}=y^{\prime}-z \in I_{m+1}, \\
Y_{m+1}=\left\{y^{\prime \prime}: y \in Y_{m}\right\} .
\end{gathered}
$$

Theorem 4.2. $p_{m}^{*}$ induces an isomorphism

$$
S_{m} /<Y_{m}>\simeq \hat{R}_{m}
$$

Proof. We know the induced map is well defined, surjective and takes $\hat{F}$ into the adic filtration. To prove injectivity it will suffice to show $p_{m}^{*}$ induces an isomorphism, for each i,

$$
g r^{i}\left(\hat{p}_{m}^{*}\right): g r^{i}\left(S_{m} /<Y_{m}>\right) \rightarrow g r^{i}(\hat{R})=S^{i}\left(\hat{T}^{1 *}\right)
$$

Given $a \in \hat{F}^{i} S_{m}$, it is clear that we may find $b \in S^{i}(H)$ with $a-b \in<Y_{m}>$. But note the exact diagram

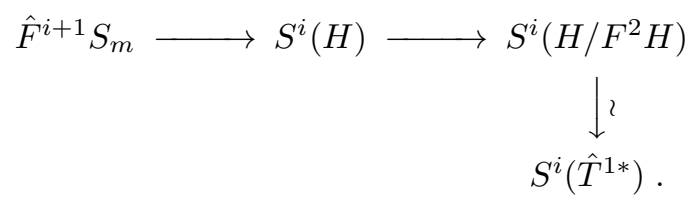

Thus if $g r^{i}\left(\hat{p}_{m}^{*}\right)(a)=0$ then $g r^{i}\left(\hat{p}_{m}^{*}(b)\right)=0$. So $b \in \hat{F}^{i+1}\left(S_{m}\right)$. Hence $a=b=0$ in $\operatorname{gr}^{i}\left(S_{m} /<Y_{m}>\right)$. 
With more specifically transcendental considerations, we shall next give more explicit versions of the above results. These involve a certain 'Green-Green' pairing \#, obtained by combining the Yukawa pairing * with the Green's operator $G$ on $X$. We now proceed to define \#.

Let $\Phi^{-1} \in H^{0}\left(\wedge^{n} T\right)$ denote the section dual to the $n$-form $\Phi$, i.e.

$$
i_{\Phi^{-1}}(\Phi)=1
$$

For forms $\alpha_{i} \in A^{n_{i}}(X), i=1,2, \alpha_{1} \otimes \alpha_{2}$ may be considered as a tensor of degree $n_{1}+n_{2}$, whence a tensor $i_{\Phi^{-1}}\left(\alpha_{1} \otimes \alpha_{2}\right)$ of degree $n_{1}+n_{2}-n$ and the Yukawa product $\alpha_{1} * \alpha_{2}$ is by definition the alternation (skew-symmetrization) of $i_{\Phi^{-1}}\left(\alpha_{1} \otimes \alpha_{2}\right)$. Note that

$$
\begin{gathered}
A^{i_{1}, j_{1}} * A^{i_{2}, j_{2}} \subseteq A^{i_{1}+i_{2}-n, j_{1}+j_{2}}, \\
\bar{\partial}\left(\alpha_{1} * \alpha_{2}\right)=\bar{\partial} \alpha_{1} * \alpha_{2} \pm \alpha_{1} * \bar{\partial} \alpha_{2},
\end{gathered}
$$

and in particular $\alpha_{1} * \alpha_{2}$ is $\bar{\partial}$-closed provided $\alpha_{1}$ and $\alpha_{2}$ are. Also note that $*$ is defined by local and holomorphic data, and makes sense for the appropriate sheaves (both holomorphic and $C^{\infty}$ ).

Now fixing a Kähler metric on $X$ (e.g. the Ricci-flat one), let $G=G_{\bar{\partial}}=G_{\partial}=$ $\frac{1}{2} G_{d}$ be the Green's operator on $X$ (cf. $\left.[\mathrm{GH}]\right)$. Thus

$$
\left(\bar{\partial} \bar{\partial}^{*}+\bar{\partial}^{*} \bar{\partial}\right) G(c)=c
$$

whenever $c$ is $d-$ or $\partial-$ or $\bar{\partial}$-exact. In fact in this case we have by the Hodge identities

$$
c=\sqrt{-1} \bar{\partial} \partial \Lambda G(c)
$$


where $\Lambda$ is the usual 'dual Lefschetz' operator. We define

$$
a \# b=\sqrt{-1} \partial \Lambda G \partial(a * b) .
$$

It is immediate that

$$
\begin{gathered}
A^{i_{1}, j_{1}} \# A^{i_{2}, j_{2}} \subseteq A^{i_{1}+i_{2}-n+1, j_{1}+j_{2}-1} \\
\bar{\partial}(a \# b)=\partial(a * b), \partial(a \# b)=0 .
\end{gathered}
$$

Now let us identify the tangent sheaf $T$ with $\Omega^{n-1}$ via interior multiplication, by $\Phi$, and note that the subsheaf $\hat{T} \subset T$ thus corresponds to the subsheaf of closed forms $\hat{\Omega}^{n-1} \subset \Omega^{n-1}$. The following identity on $\hat{T}$ is easy to prove but crucial

$$
[a, b]=\partial(a * b)
$$

Here $a, b$ can be holomorphic sections of $\hat{T}$ or $\hat{T}$-valued $(0, k)$ forms, i.e. $\partial$-closed $(n-1, k)$-forms. The case $k=1$, i.e. $a, b \in A^{n-1,1}$, is essentially equivalent to the 'Tian-Todorov Lemma'; note that in this case

$$
a * b=i_{a \wedge b}(\Phi) .
$$

[We prove (4.2) for $k=0$ as the general case is similar.Write locally $\Phi=d z_{1} \wedge \ldots \wedge$ $d z_{n}, e_{i}=\partial / \partial z_{i}, a=\sum f_{i} e_{i}, b=\sum g_{j} e_{j}$ where

$$
\sum \partial f_{i} / \partial z_{i}=\sum \partial g_{j} / \partial z_{j}=0(*)
$$

by divergence- freeness. Then

$$
a * b=\sum_{i \neq j}(-1)^{i+j} f_{i} g_{j} d z_{1} \ldots \hat{d z_{i} \ldots} \hat{d}_{j} \ldots d z_{n}
$$


so $\partial(a * b)$ corresponds via $\Phi-$ multiplication to the vector field (all summations over $i \neq j$ )

$$
\begin{gathered}
\sum \partial f_{i} / \partial z_{i} g_{j} e_{j}-\sum \partial f_{i} / \partial z_{j} g_{j} e_{i} \\
-\partial g_{j} / \partial z_{j} f_{i} e_{i}+\sum \partial g_{j} / \partial z_{i} f_{i} e_{j}
\end{gathered}
$$

which by $(*)$ equals

$$
\begin{gathered}
-\sum_{j} \partial f_{j} / \partial z_{j} g_{j} e_{j}-\sum_{i \neq j} \partial f_{i} / \partial z_{j} g_{j} e_{i} \\
+\sum_{i} \partial g_{i} / \partial z_{i} f_{i} e_{i}-\sum i \neq j \partial g_{j} / \partial z_{i} f_{i} e_{j} \\
=\left[\sum f_{i} e_{i}, \sum g_{j} e_{j}\right]=[a, b]
\end{gathered}
$$

Now recall the $m$-th order period map

$$
\hat{p}_{m}^{1}: \hat{T}^{m} \rightarrow H
$$

which we describe in Theorem 4.1 as $\mathbb{H}^{0}\left(j_{m}\right)$. Note that this description implies in particular that the $k$-th graded

$$
g r^{k}\left(\hat{p}_{m}^{1}\right): S^{k} \hat{T}^{1} \rightarrow H^{n-k, k}(X)
$$

is induced by projection $\hat{T}^{1}=H^{n, 0}+H^{n-1,1} \rightarrow H^{n-1,1}$ and Yukawa multiplication $\left(H^{n-1,1}\right)^{k} \stackrel{*}{\rightarrow} H^{n-k, k}$. We refine this observation as follows.

Theorem 4.3. There is a natural splitting

$$
\hat{T}^{m}=\oplus_{1}^{m} S^{k} \hat{T}^{1}
$$


with respect to which the $k$-th component $\hat{p}_{m}^{1, k}$, i.e. the $k$-th derivative of the period map, is given by

$\hat{p}_{m}^{1, k}\left(a_{1}, \cdots, a_{k}\right)=\frac{1}{k !} \sum_{\pi \in \sum_{k}} \sum_{j=\max (n-k+1,1)}^{k}(-1)^{j-1}\left(a_{\pi(1)} \# \cdots \# a_{\pi(j)} * a_{\pi(j+1)} * \cdots * a_{\pi(k)}\right)$

where the RHS is viewed as a cocycle in $\left(A^{*}, \partial, \bar{\partial}\right)$.

Proof. The main thing will be to construct this splitting. The splittings we shall construct for various $m$ will be mutually compatible, so there is no loss of generality in assuming $k=m \geq 2$. Let $a_{1}, \ldots, a_{m} \in \hat{T}^{1}=H^{n, 0}+H^{n, 1}$. Note $\Phi$ identifies $H^{n, 0}=\mathbb{C}$. We shall construct a lifting $b$ of $a_{1} \cdots a_{m} \in S^{m}\left(\hat{\left.(T)^{1}\right)}\right.$ to $\hat{T}^{m}$. Let $a_{i}^{1}$ be the $(n-1,1)$ component of $a_{i}$. Then we may write $a_{1} \cdots a_{m}=a_{1}^{1} \cdots a_{m}^{1}+a^{\prime}$ where $a^{\prime} \in \oplus j<m S^{i}(\hat{T})$, so the list of $a^{\prime}$ is defined by induction. Thus we may assume $a_{i}=a_{i}^{1}$ are of type $(n-1,1)$ and harmonic, hence both $\partial$ - and $\bar{\partial}$-closed. Now the cohomology of $J_{m}(T)$ and $J_{m}(\hat{T})$ may be computed by formally applying suitable Schur functors to Dolbeault complexes resolving $T$ and $\hat{T}$ as in the proof of Theorem 3.1, giving rise in the case of $T$ to a double complex $\left(B^{*}, \delta, \bar{\partial}\right)$, where $\delta$ is the differential inherited from that of $J_{m}(T)$. Now we begin with

$$
b^{-m, m}=a_{1} \cdots a_{m} \in B^{-m, m}=S^{m}\left(A^{n-1,1}\right),
$$

whose horizontal differential is

$$
\delta\left(b^{-m, m}\right)=\frac{1}{m !} \sum_{\pi \in \Sigma_{m}}\left[a_{\pi(1)}, a_{\pi(2)}\right] a_{\pi(3)} \cdots a_{\pi(m)} .
$$

As the $a_{i}$ are $\partial$-closed, we have

$$
\begin{aligned}
\delta\left(b^{-m, m}\right) & =\frac{1}{m !} \sum_{\pi \in \Sigma_{m}} \partial\left(a_{\pi(1)} * a_{\pi(2)}\right) a_{\pi(3)} \cdots a_{\pi(m)} \\
& =\frac{1}{m !} \sum_{\pi} \bar{\partial}\left(a_{\pi(1)} \# a_{\pi(2)}\right) a_{\pi(3)} \cdots a_{\pi(m)}
\end{aligned}
$$


Thus a natural choice for the component of $\mathrm{b}$ in $B^{-m+1, m-1}$ is

$$
b^{-m+1, m-1}=\frac{1}{m !} \sum\left(a_{\pi(1)} \# a_{\pi(2)}\right) a_{\pi(3)} \cdots a_{\pi(m)} .
$$

With this we indeed have

$$
\bar{\partial}\left(b^{-m+1, m-1}\right)=\delta\left(b^{-m, m}\right)
$$

Next we must find a $b^{-m+2, m-2}$ with

$$
\bar{\partial}\left(b^{-m+2, m-2}\right)=\delta\left(b^{-m+1, m-1}\right)
$$

As above, we may set

$$
b^{-m+2, m-2}=\frac{1}{m !} \sum_{\pi \in \Sigma_{m}}\left(\left(a_{\pi(1)} \# a_{\pi(2)}\right) a_{\pi(3)}\right) \cdots a_{\pi(m)}
$$

Continuing in this way we obtain a hypercocycle $b^{*}$ for $B^{*}$, lifting $a_{1} \cdots a_{m}$, yielding the required splitting.

Now by theorem (4.1), it follows that (identifying as always $T$ with $\Omega^{n-1}$ ):

$$
\begin{aligned}
\hat{p}_{m}^{1}(b) & =\sum_{j=1}^{m} i_{b^{-m+j, m-j}}(\Phi) \\
& =\frac{1}{m !} \sum_{\bar{n}} \sum_{j=1}^{m} i_{\left(a_{\pi(1)} \# \cdots \# a_{\pi(j)}\right) \wedge a_{\pi(j+1)} \wedge \cdots \wedge a_{\pi(m)}}(\Phi) \\
& =\frac{1}{m !} \sum_{\pi} \sum_{m-j+1 \leq n}\left(a_{\pi(1)} \# \cdots \# a_{\pi(j)}\right) * a_{\pi(j+1)} * \cdots * a_{\pi(m)}
\end{aligned}
$$

proving (4.3).

Using (4.3), we can give a more explicit construction of the Schottky relations $Y_{m}$. Let

$$
\eta^{i *}=\gamma^{i}: A^{i, n-i}=A^{n-i, i *} \rightarrow S^{i}\left(A^{n-1,1}\right)^{*}=S^{i}\left(A^{1, n-1}\right)
$$


be the 'Yukawa comultiplication', and likewise

$$
\rho^{j}: A^{1, n-1} \rightarrow S^{j}\left(A^{1, n-1}\right)
$$

be the Green-Green comultiplication, dual to the \# multiplication; we may extend $\rho^{j}$ as a derivation to a map

$$
\rho^{i, j}: S^{i}\left(A^{1, n-1}\right) \rightarrow S^{i+j-1}\left(A^{1, n-1}\right) .
$$

(i.e. acting on one factor at a time). Then define

$$
\nu^{i, j}: A^{i, n-i} \rightarrow S^{i+j-1}\left(H^{1, n-1}\right)
$$

as the harmonic projection of $\rho^{i, j} \circ \gamma^{i}$. (Note that $\left.H^{1, n-1}=\left(H^{n-1,1}\right)^{*}\right)$. It then follows directly from (4.3) and the above recipe for $Y_{m}$ that

Corollary 4.4. A complete set of $m$-th order Schottky relations for $X$ is given by

$$
\left\{a+\sum_{j=1}^{m}(-1)^{j} \nu^{i, j}(a): a \in H^{i, n-i} \cdot i=2, \cdots, n\right\}
$$

Remark 4.5. Unfortunately the Green's operator $G$ and its relatives, having to do with explicit realizations of the cohomology, are notoriously difficult to compute explictly, except in some special cases, e.g. curves. On the other hand it is possible to work with $\breve{C}$ ech cohomology where an explicit analogue of $G$ has been constructed in the work of Toledo and Tong [TT]. This looks like a promising way to write down period maps fairly explicitly in general cases. We hope to return to this elsewhere. See $[\mathrm{L}]$ for some explicit calculations in the curve case. 


\section{Appendix A. The Basic COnstruction, DGLA CASE}

Lie algebras are important in deformation theory because the symmetries of objects to be deformed generally form a continuous group whose tangent space $(=\{$ elements infinitely near identity $\})$ is a Lie algebra and it is these symmetries that are used in regluing pieces of the original object to form the deformation. Deformation problems of this type may be called 'free' or 'unconstrained'. There are however important deformation problems which are on the contrary 'constrained' or 'semitrivialized' in that some 'part' of the object to be deformed is to remain undeformed or, more accurately to deform in a trivialized-rather than just trivialmanner. The most familiar example of a semitrivialized problem is the Hilbert scheme, i.e. deformations of a submanifold $Y \subset X$, fixing $X$. The (full) Lie algebra associated to the embedding $Y \subset X$ is $T_{Y / X}$, the sheaf of vector fields on $X$ tangent to $Y$ along $Y$, and the corresponding (unconstrained) deformation problem is that of deformations of the pair $(X, Y)$. The viewpoint we adopt here is that deforming $Y$ in a fixed $X$ amounts to deforming the pair $(X, Y)$ and trivializing the $X$ part. In the general case this viewpoint leads to a (special kind of ) differential graded Lie algebra (DGLA), essentially consisting in a Lie algebra $g$, a $g$-module $h$ and a derivation $g \rightarrow h$; roughly $g$ will do the deforming and $h$ the trivialising. In the Hilbert scheme case $h=T_{X}$, of course, but it is important to observe that the Lie algebra structure of $T_{X}$ itself plays no role, only the $T_{X / Y}$-module structure. A succinct way to describe the situation in this case in terms of the normal sheaf $N=T_{X} / T_{X / Y}$ is that $N[-1]$ forms a 'Lie algebra in the derived category' controlling the deformations of $Y$ in the fixed $X$. 
Now recall that by definition a $(0,1)$-DGLA consists of a Lie algebra $g$, a $g$ module $h$ plus a Lie derivation $d: g \rightarrow h$; as we shall consider no other kind of DGLA, we shall for convenience drop the $(0,1)$ tag. Note that the universal enveloping algebra $U(g \rightarrow h)$ is a differential graded associative algebra, with elements of $h$ having degree 1 and the differential being the natural extension of $d$ as internal derivation of degree +1 , Note that the Lie 'inclusion' $g \rightarrow(g \rightarrow h)$ induces a homomorphism $U(g) \rightarrow U(g \rightarrow h)$ and in view of the rule

$$
b . a=a . b-a(b), a \in U(g), b \in S \cdot(h),
$$

$a(b)$ being the action of $a$ on $b$, it is easy to see that as $U(g)$-modules, we have

$$
U(g \rightarrow h) \simeq U(g) \otimes S^{\cdot}(h) .
$$

Note that to a DGLA sheaf $g \rightarrow^{d} h$ on $X$ we may associate Jacobi bicomplexes $J_{m}(g \rightarrow h)$ whose $j$ th row is of the form $\pi_{m-j, j *} J_{m-j}\left(g, \sigma^{j} h\right)$ where $\pi_{m-j, j}: X<$ $m-j, j>\rightarrow X<m>$ is the natural (forgetful) map, zeroth row is just $J_{m}(g)$ and whose $k$ th column is an Eagon-Northcott type complex of the form

$$
\lambda^{k} g \rightarrow \ldots \lambda^{j} g \otimes \sigma^{k-j} h \rightarrow \ldots \sigma^{k} h
$$

it is the derivation property of $d$ that ensures that these fit together to form a bicomplex. As in the Lie algebra case, $\mathbb{H}^{0}\left(J_{m}(g \rightarrow h)\right)$ is an OS structure with a natural OS morphism $\mathbb{H}^{0}\left(J_{m}(g \rightarrow h)\right) \rightarrow \mathbb{H}^{0}\left(J_{m}(g)\right)$, inducing a ring homomorphism $R_{m}(g) \rightarrow R_{m}(g \rightarrow h)$.

Remark A.1. For cohomological purposes we may replace $g \rightarrow h$ by a $\breve{C}$ ech bicomplex, thus turning $J_{m}(g \rightarrow h)$ into a multicomplex $\left\{\wedge^{a} C^{b}(g) \otimes S^{c} C^{d}(h)\right\}($ where $\wedge$ and $S$ are to be understood in the graded sense). The contribution to $\mathbb{H}^{0}$ 
from the 'pure $h$ ' part $a=b=0$ may be identified with the kernel of the differential $\tilde{d}$ induced by $d$ on the enveloping algebra of the DGLA $C^{\cdot}(g) \rightarrow C^{\cdot}(h)$. Moreover, in cases of interest to us, we have that $H^{0}(g) \rightarrow H^{0}(h)$ is injective and in this case it is easy to check that there is no other contribution to $\mathbb{H}^{0}(J)$, so in fact

$$
\mathbb{H}^{0}\left(J_{m}(g \rightarrow h)\right)=\operatorname{coker}(\tilde{d})
$$

Now our purpose is to interpret $R_{m}(g \rightarrow h)$ deformation theoretically. To this end let $E$ be a $g$-module over $X, R_{m}$ an artin local $\mathbb{C}$-algebra of exponent $m$, and $E_{m}$ a flat $g$-deformation of $E$ over $R_{m}$, i.e. an $R_{m}$-module locally isomorphic to $E \otimes R_{m}$ with gluing maps in $\operatorname{Exp}(g) \otimes R_{m}=G_{m}$. As in [R2], $E_{m}$ comes from a Kodaira-Spencer homomorphism $\alpha_{m}: R_{m}(g) \rightarrow R_{m}$, i.e. $E_{m} \simeq \alpha_{m}^{*} E_{m}^{u}$ where $E_{m}^{u}$ is the $m$-universal object. Now $h$ itself being a $g$-module, $\alpha_{m}$ similarly gives rise to a $g$-deformation $h_{m}=\alpha_{m}^{*} h_{m}^{u}$ where $h_{m}^{u}=M_{m}(g, h)=\operatorname{Hom}_{M O S}\left(V_{0}^{m}, \mathbb{R}^{0} p_{*}\left(J_{m}(g, h)\right.\right.$ (Theorem 3.1)(note objects like $h_{m}^{u}$ appear there without the 'u' superscript); note that $m_{m} h_{m}^{u}=\operatorname{Hom}_{M O S}\left(V_{m}, \mathbb{R}^{0} p_{*}\left(J_{m}(g, h)\right.\right.$. Now the canonical map $J_{m}(g) \rightarrow$ $\pi_{m, 1 *} J_{m}(g, h)[1]$, which is nothing but the map from the zeroth to the first row of $J_{m}(g \rightarrow h)$, gives rise to a canonical cohomology class $B_{m}(h) \in C^{m} \mathbb{H}^{1}\left(J_{m}(g, h)\right)=$ $H^{1}\left(h_{m}^{u}\right)$ (in fact this clearly lifts to $H^{1}\left(m_{m} h_{m}^{u}\right)$ and we shall use $B_{m}(h)$ to denote the lift as well), whence a class

$$
B\left(\alpha_{m}, h\right)=\alpha_{m}^{*}\left(B_{m}(h)\right) \in H^{1}\left(m_{m} h_{m}^{u}\right)
$$

Note incidentally that $g$ itself is a $g$-module via the $1 / 2$ ad action and for this action $g \rightarrow^{i d} g$ is a DGLA (the $1 / 2$ factor is needed to make $i d$ a derivation), whence a canonical element $B\left(\alpha_{m}, g\right) \in H^{1}\left(m_{m} g_{m}\right)$. For instance if $g=T_{X}$ then $g_{m}=$ $T_{X_{m} / R_{m}}$, the relative tangent sheaf. 
By a $(g \rightarrow h)$-deformation of $E$ we mean the data $\left(\alpha_{m}, A\right)$ consisting of a $g$ deformation of $E$ given by $\alpha_{m}$ plus a 'trivialization' of the corresponding canonical cohomology class, given e.g. by a $\check{C}$ ech cochain $A=\left(v_{\alpha}\right) \in \check{C}^{\circ}\left(h_{m}\right), \delta A=$ $B\left(\alpha_{m}, h\right)$. These objects can all be represented concretely as in [R2]. Recall to begin with that $\alpha_{m}$ corresponds to a morphic hypercocycle $v_{m}=\epsilon\left(u_{m}\right) \in \mathbb{H}^{0}\left(J_{m}(g)\right) \otimes m_{m}$ where $u .=u_{m} \in \breve{C}^{1}(g) \otimes m_{m}$ satisfies the $\breve{C}$ ech integrability condition

$$
\delta\left(u_{m}\right)=-1 / 2\left[u_{m}, u_{m}\right]
$$

a deformation corresponding to $\alpha_{m}$, such as $E_{m}$ (or $g_{m}$ or $h_{m}$ ) is essentially defined by the condition that its $\breve{C}$ ech complex $\left(\breve{C} \cdot\left(E_{m}\right), \delta_{m}\right)$ be isomorphic to $(\breve{C} \cdot(E) \otimes$ $R_{m}, \delta_{0} \otimes 1+u_{m}$ ) (viewing $u_{m}$ as an operator of degree +1 on $\left.\breve{C} \cdot\left(E_{m}\right) \otimes R_{m}\right)$. Then $B\left(\alpha_{m}, g\right)$ is represented by $\left(u_{m}\right)$ (which by (A.2) is indeed a cocycle for $g_{m}$ and in fact for $m_{m} g_{m}$, which is a flat $R_{m-1}$-module); similarly, $B\left(\alpha_{m}, h\right)$ is represented by $d\left(u_{m}\right)$. Put another way, the operator $\delta_{0} \otimes 1$ on $\left.\check{C}^{(} E\right) \otimes R_{m}$ pulled over to $\check{C} \cdot\left(E_{m}\right)$ becomes $\delta-u_{m}$, so $u_{m}$ is indeed a cocycle for $m_{m} g_{m}$. Consequently $A$ may be represented by

$$
\begin{gathered}
a . \in \breve{C}^{0}(h) \otimes m_{m}, \\
\delta(a .)+u .(a .)=d(u .)
\end{gathered}
$$

i.e. $a_{\alpha}-a_{\beta}+1 / 2 u_{\alpha \beta}\left(a_{\alpha}+a_{\beta}\right)=d\left(u_{\alpha \beta}\right)$. The following then is the DGLA analogue of the main result of [R2].

Theorem A.2. Assume $H^{0}(g) \rightarrow H^{0}(h)$ is injective. Then for any $m$, there exists a $(g \rightarrow h)$ deformation $\left(\alpha_{m}^{u c}, A_{m}^{u c}\right)$ over $R_{m}^{u c}=R_{m}(g \rightarrow h)$ which is constrained 
universal in that for any $(g \rightarrow h)$ deformation $\left(\alpha_{m}, A_{m}\right)$ over $R_{m}$, there is a factorization of $\alpha_{m}$

$$
R_{m}^{u} \stackrel{\alpha_{m}^{u c}}{\rightarrow} R_{m}^{u c} \stackrel{\beta_{m}}{\rightarrow} R_{m}
$$

such that $A_{m}=\beta_{m}^{*} A_{m}^{u c}$.

Proof. Analogous, mutatis mutandis, to that in [R2] (compare Theorem 3.1 above). To begin with, $\alpha_{m}^{u c}$ simply corresponds to the natural map $q_{m}: J_{m}(g \rightarrow h) \rightarrow$ $J_{m}(g)$, while $A_{m}^{u c}$ comes from the extra data needed to lift an element of $\mathbb{H}^{0}\left(J_{m}(g)\right)$ to one in $\mathbb{H}^{0}$ of the double complex formed from the last 2 rows of $J_{m}(g \rightarrow h)$. Now given an arbitrary $(g \rightarrow h)$-deformation $\left(\alpha_{m}, A_{m}\right)$ over $R_{m}, \alpha_{m}$ corresponds to a cochain $u_{m} \in C^{1}(g) \otimes m_{m}$ and $A_{m}$ to $a \in C^{0}(h) \otimes m_{m}$ as above. Then

$\left(u_{m}, \frac{1}{2} u_{m}^{2}, \cdots, \frac{1}{m !} u_{m}^{m} ; a, u_{m} \times a, \cdots, \frac{1}{(m-1) !} u_{m}^{m-1} \times a ; \frac{1}{2} a^{2}, u_{m} \times \frac{1}{2} a^{2} ; \cdots ; \cdots ; \frac{1}{m !} a^{m}\right)$

yields a morphic element of $m_{m} \otimes \mathbb{H}^{0}\left(J_{m}(g \rightarrow h)\right)$ corresponding to $\beta_{m}$ as required.

\section{Examples}

1. Let $X$ be a compact complex complex manifold and $Y \subset X$ a submanifold with normal bundle $N$. We have a DGLA

$$
\left(T_{X / Y} \rightarrow T_{X}\right) \sim N[-1]
$$

As noted before $([\mathrm{R} 2]$,section 5$), T_{X / Y}$ controls deformations $\left(Y_{m} \subset X_{m} / R_{m}\right)$ of the pair $Y \subset X$ and given such a deformation with Kodaira-Spencer homomorphism $\alpha_{m}: R_{m}\left(T_{X / Y}\right) \rightarrow R_{m}$, evidently $B\left(\alpha_{m}, T_{X}\right)$ is just the class corresponding to the 
induced deformation $X_{m}$ of $X$ alone, so that the data $A$ is simply an identification of $X_{m}$ with the trivial deformation $X \times \operatorname{Spec}\left(R_{m}\right)$.

Remark A.3. The cokernel of $\tilde{d}$ on $U(N[-1])$ is a certain quotient of $S \cdot\left(T_{X}\right)$ which is essentially the sheaf $D_{Y \rightarrow X}$ of 'normal differential operators' along $Y$, considered by Burchard, Clemens et al $[\mathrm{BC}]$. Note the natural map

$$
\begin{aligned}
\mathbb{H}^{0}\left(J_{m}(N[-1])\right)=\operatorname{coker}\left(\tilde{d}, \mathbb{H}^{0}\right) & \rightarrow \mathbb{H}^{0}(\operatorname{coker}(\tilde{d}, U(N[-1]))) \\
& \rightarrow \mathbb{H}^{0}\left(D_{Y \rightarrow X}\right)
\end{aligned}
$$

2. (cf.[R3]) Let X be a compact complex Kähler manifold with tangent sheaf $T$ and $\eta \in H^{p, q}(X)$. We have a map

$$
T \rightarrow \Omega^{p-1}[q]
$$

given by interior multiplication by $\eta$. Representing $\eta$ by a closed form, note the formula

$$
i_{[x, y]}(\eta)=L_{x}\left(i_{y}(\eta)\right)-L_{y}\left(i_{x}(\eta)\right)-d\left(i_{x \wedge y}(\eta)\right)
$$

for vector fields $x, y$. Replacing $\Omega^{p-1}$ by, e.g. its Dolbeault resolution we have by the usual Kähler machinery

$$
d\left(i_{x \wedge y}(\eta)\right)=\bar{\partial}\left(j_{x \wedge y}(\eta)\right)
$$

for a suitable well-defined $j_{x \wedge y}(\eta) \in A^{p-1, q-1}$, therefore this term vanishes in the derived category and $\mathcal{L}_{\eta}=\left(T \rightarrow \Omega^{p-1}[q]\right)$ forms a DGLA. As shown in [R3], $\mathcal{L}_{\eta}$ controls precisely the deformations of $\mathrm{X}$ in which (the GM-constant lift of) $\eta$ maintains Hodge level $p$. In the situation of example 1, we may take $\eta=[Y] \in$ 
$H^{p, p}(X), p=\operatorname{codim}(Y)$ and then we have an exact diagram

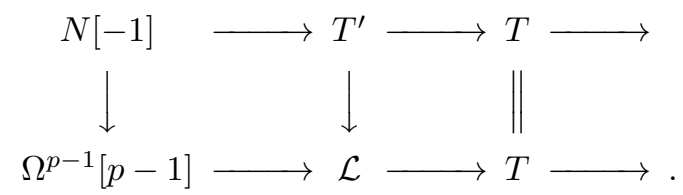

See [R3] for geometric application of this.

\section{REFERENCES}

[BC] P. Burchard, H. Clemens: pers. comm. from H. Clemens.

[C] H. Clemens: 'Cohomology and obstructions', preprint.

[D] P. Deligne:'Theoreme de Lefschetz et criteres de degenerescence de suites spectrales'. Pub;. Math. IHES 35 (1968),197-226.

[FH] W. Fulton, J. Harris: 'Introduction to Representation Theory.' Springer.

[GH] P. Griffiths, J. Harris: 'Principles of Algebraic Geometry' Wiley 1977.

[EV] H. Esnault, E. Viehweg :'Higher Kodaira-Spencer classes' Math. Annalen 299 491-527 (1994).

[K] N. Katz :' Algebraic solutions of differential equations ' Invent. math 18 1-118 (1972).

[L] G. Liu :UCR dissertaion, 1997.

[G] P. Griffiths,:'Topics in transcendental algebraic geometry'. Ann. of Math. Studies. Princeton 1986.

[R1] Z. Ran, :'Derivatives of moduli.' Int. math. res. notices (1993), 93-106.

[R2] Z. Ran, :'Canonical inginitesimal deformations ', Preprint.

[R3] Z. Ran, :'Semiregularity mappings and obstructions', Preprint.

[TT] D. Toledo, Y. Tong.:'A parametrix for $\bar{\partial}$ and Riemann-Roch in Cech theory.' Topology 15, 273-301 1976 . 